\title{
Hypergeometric forms for Ising-class integrals
}

\author{
D.H. Bailey*, \\ D. Borwein \\ J.M. Borwein $\ddagger$ \\ R.E. Crandall ${ }^{\S}$
}

September 11, 2006

Abstract: We apply experimental-mathematical principles to analyze integrals

$$
C_{n, k}:=\frac{1}{n !} \int_{0}^{\infty} \cdots \int_{0}^{\infty} \frac{d x_{1} d x_{2} \cdots d x_{n}}{\left(\cosh x_{1}+\cdots+\cosh x_{n}\right)^{k+1}} .
$$

These are generalizations of a previous integral $C_{n}:=C_{n, 1}$ relevant to the Ising theory of solid-state physics [8]. We find representations of the $C_{n, k}$ in terms of Meijer $G$-functions and nested-Barnes integrals. Our investigations began by computing 500-digit numerical values of $C_{n, k}$ for all integers $n, k$ where $n \in[2,12]$ and $k \in[0,25]$. We found that some $C_{n, k}$ enjoy exact evaluations involving Dirichlet $L$-functions or the Riemann zeta function. In the process of analyzing hypergeometric representations, we found - experimentally and strikingly - that the $C_{n, k}$ almost certainly satisfy certain inter-indicial relations including discrete $k$-recursions. Using generating functions, differential theory, complex analysis, and Wilf-Zeilberger algorithms we are able to prove some central cases of these relations.

\footnotetext{
*Lawrence Berkeley National Laboratory, Berkeley, CA 94720, dhbailey@lbl.gov. Supported in part by the Director, Office of Computational and Technology Research, Division of Mathematical, Information, and Computational Sciences of the U.S. Department of Energy, under contract number DE-AC02-05CH11231.

${ }^{\dagger}$ Department of Mathematics University of Western Ontario, London, ONT, N6A 5B7 , Canada, dborwein@uwo.ca. Supported in part by NSERC.

${ }^{\ddagger}$ Faculty of Computer Science, Dalhousie University, Halifax, NS, B3H 2W5, Canada, jborwein@cs.dal.ca. Supported in part by NSERC and the Canada Research Chair Programme.

$\S$ Center for Advanced Computation, Reed College, Portland OR, crandall@reed.edu.
} 


\section{Background and nomenclature}

The primary entities on which the present work will focus are the $n$-dimensional integrals

$$
C_{n, k}:=\frac{1}{n !} \int_{-\infty}^{\infty} \cdots \int_{-\infty}^{\infty} \frac{d x_{1} d x_{2} \cdots d x_{n}}{\left(\cosh x_{1}+\cdots+\cosh x_{n}\right)^{k+1}}
$$

These integrals are well defined - in fact absolutely convergent - for any positive integer $n$ and any complex $k \in \mathcal{K}$ where we speak of the open half-plane

$$
\mathcal{K}:=(z \in \mathcal{C}: \Re(z)>-1) .
$$

The integrals $C_{n, k}$ can be traced back to the Ising theory of solid-state physics. As summarized in a previous work [8], there is interest in giving closed forms and growth bounds for $n$-dimensional Ising susceptibility integrals

$$
D_{n}:=\frac{4}{n !} \int_{0}^{\infty} \cdots \int_{0}^{\infty} \frac{\prod_{i<j}\left(\frac{u_{i}-u_{j}}{u_{i}+u_{j}}\right)^{2}}{\left(\sum_{j=1}^{n}\left(u_{j}+1 / u_{j}\right)\right)^{2}} \frac{d u_{1}}{u_{1}} \cdots \frac{d u_{n}}{u_{n}} .
$$

These $D_{n}$ appear-with various normalizations - in the standard Ising literature $[29,30,40,41,42,43]$. The quest for closed forms for Ising susceptibility integrals thus led to a definition in [8] of a class of structurally similar integrals, among which is the structure (2) but without the permutation product in the integrand, namely

$$
C_{n}:=\frac{4}{n !} \int_{0}^{\infty} \cdots \int_{0}^{\infty} \frac{1}{\left(\sum_{j=1}^{n}\left(u_{j}+1 / u_{j}\right)\right)^{2}} \frac{d u_{1}}{u_{1}} \cdots \frac{d u_{n}}{u_{n}},
$$

which, as can be seen via a transformation $u_{k} \rightarrow e^{x_{k}}$ is the case $C_{n, 1}$ of the key definition (1).

A brief digression here is worthwhile. There is an even more general class of integrals that likewise admit of analytical promise. We may define, for integer $n$, complex $k$, and an $n$-vector $\vec{r}:=\left(r_{1}, \ldots, r_{n}\right)$ of complex numbers the entities

$$
C_{n, k, \vec{r}}:=\frac{1}{n !} \int_{-\infty}^{\infty} \cdots \int_{-\infty}^{\infty} \frac{\prod_{j=1}^{n} \cosh \left(r_{j} x_{j}\right)}{\left(\cosh x_{1}+\cdots+\cosh x_{n}\right)^{k+1}} d x_{1} \cdots d x_{n} .
$$

Absolute convergence of the integral is assured on the condition that $k$ lie in the translated half-plane $\mathcal{K}+\Re\left(\sum r_{j}\right)$. Thus we can restrict indices to obtain integrals of our primary interest, e.g.

$$
\begin{aligned}
C_{n, k} & :=C_{n, k, \overrightarrow{0}}, \\
C_{n} & :=C_{n, 1}:=C_{n, 1, \overrightarrow{0}} .
\end{aligned}
$$

One reason to contemplate these generalized $C_{n, k, \vec{r}}$ is that they enjoy certain combinatorial relations when cast in so-called Bessel-kernel form, as we shall 
later see in Section 7. In principle, one could also allow continuous $n$, and so a prefactor $1 / \Gamma(n+1)$, with a fractional-dimensional integral defined in Besselkernel terms; so there could be yet more useful generalization. We will sometimes write $n$ ! for the analytic quantity $\Gamma(n+1)$.

An outline of the paper is as follows. In $\S 2$ we examine hypergeometric and related expressions for our integrals. Then in $\S 3$ we describe closed forms and series for individual $C_{n, k}$. In $\S 4$ and $\S 5$ we explore recursion relations. In $\S 6$ related continued fractions are given, while in $\S 7$ we explore further analytic properties of the $C_{n, k}$. Finally, in $\S 8$ we discuss our extreme-precision numerics before concluding with some open problems.

\section{Hypergeometric connections}

It turns out that the Ising-class integrals $C_{n, k}$ enjoy certain connections with hypergeometric functions and their powerful generalization, the Meijer G-functions. Such analysis gives rise to fascinating series representations, new closed forms, and rational relations between certain pairs of integrals. (We refer the reader also to our separate work on the quest for closed Ising forms [8].) Not surprisingly, the collection $\left(C_{n, k}: n \in \mathbb{Z}^{+}, k \in \mathcal{K}\right)$ provides fertile ground for experimental-mathematical discovery, not to mention clues as to what symbolic behavior might be expected of Ising integrals in general. In addition, we derive some evidently new exact evaluations of Meijer $G$-functions themselves.

A Bessel-kernel representation we developed in [8] likewise generalizes to

$$
C_{n, k}=\frac{2^{n}}{n !} \frac{1}{\Gamma(k+1)} c_{n, k},
$$

where we use $\Gamma(k+1)=k$ ! to emphasize that $k$ need not be integer, and where the (lowercase) $c$ definition is

$$
c_{n, k}:=\int_{0}^{\infty} t^{k} K_{0}(t)^{n} d t .
$$

(here $K_{0}$ is the modified Bessel function). This representation, as in [8], permits us to calculate explicit values to very high precision (our 500-digit values are available online [9]). Note that in regard to $k$-dependence $c_{n, k}$ differs from $C_{n, k}$ by a prefactor of $\Gamma(k+1)$; this scaling will be convenient later, when we analyze recurrence relations.

It is clear from the definition (1) that for fixed integer $n, C_{n, k}$ is monotonic decreasing in real $k$. The arguments of theorems in [8] regarding the original $C_{n}$ can be augmented to show first, that for fixed real $k \geq 1$ the set $\left(C_{n, k}\right)$ is monotonic decreasing in $n$, and that for any fixed $k$ we have the large- $n$ asymptote

$$
C_{n, k} \sim \frac{1}{\Gamma(k+1)} \frac{2^{k+1+n}}{(k+1)^{n+1}} e^{-(k+1) \gamma},
$$


for which our original, canonical case in [8] reads $C_{n}=C_{n, 1} \sim_{n} 2 e^{-2 \gamma} \approx$ $0.63047 \ldots$ This asymptotic behavior is revealed by extreme-precision numerical values for $C_{n}$. An example of the data downloadable at [9] is the following, where the asymptote $2 e^{-2 \gamma}$ is evident:

\begin{tabular}{rc}
$n$ & $C_{n}$ \\
\hline 4 & $0.70119986017642999981651392754834582794624200386529 \ldots$ \\
16 & $0.63050394617323726350529565756068741948431621720810 \ldots$ \\
64 & $0.63047350337438679648836208816533862535998880860015 \ldots$ \\
256 & $0.63047350337438679612204019271087890435458707871273 \ldots$ \\
1024 & $0.63047350337438679612204019271087890435458707871273 \ldots$ \\
\hline
\end{tabular}

Another observation on the generalization $C_{n, k, \vec{r}}$ is in order. Some idea of the power of Bessel representation such as (7) can be gleaned by the observation that, for vector $\vec{r}:=(p, p, \ldots, p)=(\bar{p})$ we have again a 1-dimensional integral

$$
C_{n, k,(\bar{p})}:=\frac{2^{n}}{n !} \frac{1}{\Gamma(k+1)} \int_{0}^{\infty} t^{k} K_{p}(t)^{n} d t .
$$

It is interesting that for $p$ a half-odd integer, the Bessel function is elementary and we routinely obtain closed forms. For example, for general complex $k$ we infer

$$
C_{4, k,(3 / 2,3 / 2,3 / 2,3 / 2)}=\frac{2^{1-2 k} \pi^{2} \Gamma(k-5)}{3 \Gamma(k+1)}\left(k^{4}+2 k^{3}-25 k^{2}-10 k+56\right)
$$

of which an instance is

$$
C_{4,6,(\overline{3 / 2})}=103 \pi^{2} / 552960 .
$$

Though such cases do not shed much light on our main theme - the $C_{n, k}$ themselvesthese tractable cases do suggest such notions as analytic continuation (in $k$, beyond the relevant half-plane) as well as the appearance of polynomials in $k$.

We shall be analyzing series representations and closed forms for various $C_{n, k}$. To this end, we state some exact integrals based on the Adamchik algorithm described in [2]:

$$
\begin{gathered}
c_{1, k}=\int_{0}^{\infty} t^{k} K_{0}(t) d t=2^{k-1} \Gamma\left(\frac{k+1}{2}\right)^{2}, \\
c_{2, k}=\int_{0}^{\infty} t^{k} K_{0}^{2}(t) d t=\frac{\sqrt{\pi} \Gamma\left(\frac{k+1}{2}\right)^{3}}{4 \Gamma\left(\frac{k}{2}+1\right)}, \\
c_{3, k}=\int_{0}^{\infty} t^{k} K_{0}^{3}(t) d t=2^{k-2} \sqrt{\pi} G_{3,3}^{3,2}\left(4 \mid \begin{array}{c}
\frac{1-k}{2}, \frac{1-k}{2}, \frac{1}{2} \\
0,0,0
\end{array}\right),
\end{gathered}
$$


where the relevant Meijer $G$-function here is

$$
G:=\frac{1}{2 \pi i} \int_{\mathcal{C}} \frac{\Gamma^{2}((k+1) / 2-s) \Gamma^{3}(s)}{\Gamma(s+1 / 2)} 4^{-s} d s .
$$

Finally, we have

$$
c_{4, k}=\int_{0}^{\infty} t^{k} K_{0}^{4}(t) d t=\frac{1}{8} \pi G_{4,4}^{3,3}\left(\begin{array}{l|c}
1 & \begin{array}{c}
1,1,1, \frac{k+2}{2} \\
\frac{k+1}{2}, \frac{k+1}{2}, \frac{k+1}{2}, \frac{1}{2}
\end{array}
\end{array}\right),
$$

where in this case the relevant Meijer $G$-function is

$$
G:=\frac{1}{2 \pi i} \int_{\mathcal{C}} \frac{\Gamma^{3}(-s) \Gamma^{3}((k+1) / 2+s)}{\Gamma(1+k / 2+s) \Gamma(1 / 2-s)} d s
$$

In the above cases $n=3,4$ the contour $\mathcal{C}$ encompasses all poles of the first $\Gamma$ form in the numerator, but no other poles, as is consistent with formal definitions of the Meijer $G$ 's as given in $[2,32]$. In our study, said contour can always be taken as a vertical run, upward, and intersecting the real $s$-axis at an appropriate place, say $s=-1 / 2$. It is unknown how to generalize such Meijer formulae beyond the 4-th power of the Bessel- $K$-i.e., once again, as happened in the work [8], we encounter a kind of theoretical blockade for $n \geq 5$.

In spite of the blockade for $n \geq 5$ in regard to Meijer- $G$ representations, we shall still be able to represent, in our Section 7 , arbitrary $C_{n, k}$ via yet more complicated structures.

\section{Closed forms and series for individual $C_{n, k}$}

\subsection{Evaluations of $C_{1, k}$}

Immediately from relations $(7,10)$ we have

$$
C_{1, k}=\frac{2^{k} \Gamma\left(\frac{k+1}{2}\right)^{2}}{\Gamma(k+1)}
$$

The first few exact evaluations are $\left(C_{1,0}, C_{1,1}, C_{1,2}, C_{1,3}, \ldots\right)=(\pi, 2, \pi / 2,4 / 3, \ldots)$. It is evident that for any $k \geq 1$,

$$
C_{1, k}=p_{1, k}+q_{1, k} \pi
$$

where the $p, q$ coefficients are always rational, with $q$ vanishing for odd $k$ and $p$ vanishing for even $k$. This observation about the character of the $p, q$ is trivial, but as we shall eventually see, such a " $p+q x$ " pattern for larger $n$ becomes radically more profound. 


\begin{tabular}{|c|c|c|}
\hline$n$ & $k$ & $C_{n, k}$ \\
\hline 1 & any & $\frac{2^{k} \Gamma\left(\frac{k+1}{2}\right)^{2}}{k !}=p_{1, k}+q_{1, k} \pi$ \\
\hline 2 & any & $\frac{\sqrt{\pi} \Gamma\left(\frac{k+1}{2}\right)^{3}}{2 \Gamma\left(\frac{k}{2}+1\right) \Gamma(k+1)}=p_{2, k}+q_{2, k} \pi^{2}$ \\
\hline 3 & 0 & Elliptic form (21) \\
\hline 3 & 1 & $C_{3}=\mathrm{L}_{-3}(2) \quad($ see $[8])$ \\
\hline 3 & 2 & Elliptic form (24) \\
\hline 3 & 3 & $C_{3,3}=\frac{2}{9} \mathrm{~L}_{-3}(2)-\frac{4}{27}$ \\
\hline 3 & any odd & $p_{3, k}+q_{3, k} \mathrm{~L}_{-3}(2)$, Series $(16)$ \\
\hline 3 & any even & Order-2 recursion (Thm. 5), Series (17) \\
\hline 3 & any complex & Meijer integral (12) \\
\hline 4 & 0 & Series $(31)$ \\
\hline 4 & 1 & $C_{4}=\frac{7}{12} \zeta(3) \quad($ see $[8])$ \\
\hline 4 & 3 & $C_{4,3}=\frac{7}{288} \zeta(3)-\frac{1}{48}$ \\
\hline 4 & any odd & $p_{4, k}+q_{4, k} \zeta(3)$ \\
\hline 4 & any even & Order-2 recursion (Thm. 5) \\
\hline 4 & any complex & Meijer integral (13) \\
\hline 5 & any complex & Nested-Barnes integral (55), Series (58) \\
\hline large & fixed & $\sim \frac{1}{k !} \frac{2^{k+1+n}}{(k+1)^{n+1}} e^{-(k+1) \gamma}$ \\
\hline
\end{tabular}

Table 1: Proven closed forms, series, and relations for the $C_{n, k}$. Every $p$ or $q$ coefficient above is proven rational, with the $q$ having explicit finite forms. Our searches have uncovered no other closed forms, or pairwise rational relations not implicit above. Conjecture 1 gives a general recursion relation for complex $k$. 


\subsection{Evaluations of $C_{2, k}$}

Next, from relations $(7,11)$ we obtain

$$
C_{2, k}=\frac{\sqrt{\pi} \Gamma\left(\frac{k+1}{2}\right)^{3}}{2 \Gamma\left(\frac{k}{2}+1\right) \Gamma(k+1)},
$$

with the first few being $\left(C_{2,0}, C_{2,1}, C_{2,2}, C_{2,3}, \ldots\right)=\left(\pi^{2} / 2,1, \pi^{2} / 32,1 / 9, \ldots\right)$. In this $n=2$ case we have

$$
C_{2, k}=p_{2, k}+q_{2, k} \pi^{2}
$$

with the same vanishing rule on the rational $p, q$ multipliers as for $n=1$.

\subsection{Evaluations of $C_{3, k}$}

After resolving all $C_{n, k}$ for $n=1,2$ as above, the case $n=3$ on $C_{n, k}$ suddenly becomes nontrivial, yet there are various approaches that yield new insight; at the very least, new closed-form evaluations of the appropriate Meijer- $G$. Choosing a contour and performing residue calculus (we leave out the intricate details) on the Meijer- $G$ for identity (12), one may obtain quite efficient series developments. To summarize, define $\mu:=\lfloor(k-2) / 2\rfloor$ and a polynomial

$$
P_{\mu}(x):=\prod_{a=0}^{\mu}(x-a)^{2}
$$

and an alternating harmonic number

$$
H_{c}^{(-1)}:=1-\frac{1}{2}+\frac{1}{3}-\cdots \pm \frac{1}{c}
$$

with $H_{0}^{(-1)}:=0$. Then, for odd $k$, the residue calculus yields a linearly convergent series

$$
C_{3, k}=\frac{2^{k} \sqrt{\pi}}{3 ! k !} \sum_{h=\mu+1}^{\infty} \frac{P_{\mu}(h)}{4^{h}} \frac{\Gamma(h+1)}{\Gamma(h+3 / 2)}\left(H_{2 h+1}^{(-1)}-\frac{1}{2} \frac{P_{\mu}^{\prime}(h)}{P_{\mu}(h)}\right) .
$$

Similarly, for even $k$, one obtains

$$
C_{3, k}=\frac{2^{k+1} \sqrt{\pi}}{3 ! k !} \sum_{h=\mu+1}^{\infty} \frac{P_{\mu}(h)}{4^{h}} \frac{\Gamma^{3}(h+1 / 2)}{\Gamma^{3}(h+1)}\left(4 \log 2-3 H_{2 h}^{(-1)}-\frac{1}{2} \frac{P_{\mu}^{\prime}(h)}{P_{\mu}(h)}\right) .
$$

\subsubsection{The $C_{3, \text { even }}$ integrals}

Yet another surprise in the world of Ising-class integrals is that the $C_{3 \text {,even }}$ seem to be more mysterious than the $C_{3, \text { odd }}$. One way to think of this dichotomy is to observe the way that gamma functions appear in the respective series $(16$, 
17). One may employ special hypergeometric identities, which we found in Mathematica and reconfirmed in Maple, such as

$$
\begin{array}{r}
\sum_{h=0}^{\infty} \frac{\Gamma(h+1)}{\Gamma(h+3 / 2)} \sin ^{2 h} \theta=\frac{4}{\sqrt{\pi}} \frac{\theta}{\sin (2 \theta)}, \\
\sigma_{0}(\theta):=\sum_{h=0}^{\infty} \frac{\Gamma^{3}(h+1 / 2)}{\Gamma^{3}(h+1)} \sin ^{2 h} \theta=\frac{4}{\sqrt{\pi}} \mathrm{K}^{2}\left(\sin \frac{\theta}{2}\right),
\end{array}
$$

where in the second identity $\mathrm{K}(k)$ is the (complete) elliptic integral of the first kind with modulus $k .{ }^{1}$ We may also employ an integral identity

$$
4 \log 2-3 H_{2 h}^{(-1)}=\int_{0}^{1} \frac{1+3 t^{2 h}}{1+t} d t=\log 2+3 \int_{0}^{1} \frac{t^{2 h}}{1+t} d t .
$$

Putting this all together for the special case

$$
C_{3,0}=\frac{\sqrt{\pi}}{3} \sum_{h=0}^{\infty} \frac{1}{4^{h}} \frac{\Gamma^{3}(h+1 / 2)}{\Gamma^{3}(h+1)}\left(4 \log 2-3 H_{2 h}^{(-1)}\right)
$$

we arrive at the peculiar elliptic representation

$$
C_{3,0}=\frac{4}{3} \mathrm{~K}^{2}\left(\sin \frac{\pi}{12}\right) \log 2+8 \int_{0}^{\pi / 6} \frac{\mathrm{K}^{2}\left(\sin \frac{\theta}{2}\right) \cos \theta}{1+2 \sin \theta} d \theta .
$$

Moreover

$$
\mathrm{K}^{2}\left(\sin \frac{\pi}{12}\right)=\frac{2}{27} \frac{\sqrt{3} \sqrt[3]{2} \pi^{4}}{\Gamma^{6}(2 / 3)}=\frac{\sqrt[3]{2} \sqrt{3}}{24} \beta^{2}\left(\frac{1}{3}, \frac{1}{3}\right)
$$

is the integral at the third singular value, $k_{3}[16]$. Correspondingly, the Clausen product identity $[15$, p. 50$]$ shows

$$
8 \int_{0}^{\pi / 6} \frac{\mathrm{K}^{2}\left(\sin \frac{\theta}{2}\right) \cos \theta}{1+2 \sin \theta} d \theta=\pi^{2} \int_{0}^{1}{ }_{3} \mathrm{~F}_{2}\left(\begin{array}{c}
1 / 2,1 / 2,1 / 2 \\
1,1
\end{array} ; \frac{x^{2}}{4}\right) \frac{d x}{x+1} .
$$

This elliptic-cum-hypergeometric form is a rather erudite result for the relatively innocent-looking integral

$$
C_{3,0}:=\frac{1}{6} \int_{R^{3}} \frac{d x d y d z}{\cosh x+\cosh y+\cosh z} .
$$

There are other attractive representations equivalent to the elliptic form (21) such as

$$
C_{3,0}=\pi \int_{0}^{\infty} \int_{0}^{\infty} \frac{1}{\sqrt{x^{2}+1} \sqrt{y^{2}+1} \sqrt{(x+y)^{2}+1}} d x d y
$$

\footnotetext{
${ }^{1}$ Here we use the convention $\mathrm{K}(k)=\int_{0}^{\pi / 2}\left(1-k^{2} \sin ^{2} s\right)^{-1 / 2} d s$. See [15, pg 199-200]. One should beware: Some symbolic systems use $m:=k^{2}$ as the argument; for example in Mathematica one has EllipticK $[\mathrm{m}]:=\mathrm{K}(\sqrt{m})$.
} 
We next observe that $C_{3,2}$ possesses a corresponding closed form which also involves the elliptic integral of the second kind $\mathrm{E}\left(k_{3}\right),[18]$. This may be similarly derived from (17) as follows.

Since $P_{0}(x)=x^{2}$, the building blocks for $C_{3,2}$ are

$$
\begin{aligned}
\sigma_{1}(\theta) & :=\sum_{h=0}^{\infty} \frac{h \Gamma^{3}(h+1 / 2)}{\Gamma^{3}(h+1)} \sin ^{2 h} \theta \\
& =\frac{4}{\sqrt{\pi} \cos \theta}\left\{(\mathrm{E} \mathrm{K})\left(\sin \frac{\theta}{2}\right)-\left(\cos ^{2} \frac{\theta}{2}\right) \mathrm{K}^{2}\left(\sin \frac{\theta}{2}\right)\right\}
\end{aligned}
$$

and

$$
\begin{aligned}
\sqrt{\pi} \sigma_{2}(\theta) & :=\sqrt{\pi} \sum_{h=0}^{\infty} \frac{h^{2} \Gamma^{3}(h+1 / 2)}{\Gamma^{3}(h+1)} \sin ^{2 h} \theta \\
& =\frac{(\cos \theta+1)\left(\cos ^{2} \theta+\cos \theta-1\right)}{\cos ^{3} \theta} \mathrm{K}^{2}\left(\sin \frac{\theta}{2}\right) \\
& -2 \frac{(\cos \theta+1)(2 \cos \theta-1)}{\cos ^{3} \theta}(\mathrm{E} \mathrm{K})\left(\sin \frac{\theta}{2}\right)+\frac{2}{\cos ^{2} \theta} \mathrm{E}^{2}\left(\sin \frac{\theta}{2}\right) .
\end{aligned}
$$

Thus, we may use (17) to write

$$
C_{3,2}=\frac{2 \log 2}{3} \sqrt{\pi} \sigma_{2}\left(\frac{\pi}{6}\right)-\frac{2}{3} \sqrt{\pi} \sigma_{1}\left(\frac{\pi}{6}\right)+4 \int_{0}^{\pi / 6} \sqrt{\pi} \sigma_{2}(\theta) \frac{\cos \theta}{1+2 \sin \theta} d \theta .
$$

Also, for $\theta=\pi / 6$, we have EK $=\left(\pi+(2+2 \sqrt{3}) \mathrm{K}^{2}\right) \sqrt{3}$, see [18]. Thus, using (24) we will get two more-complicated terms like the ones in $C_{3,0}$ but now involving both $\mathrm{E}$ and $\mathrm{K}$. Note that $\cos \pi / 12=(\sqrt{3}+1) / \sqrt{8}$ and $\sin \pi / 12=$ $(\sqrt{3}-1) / \sqrt{8}$ are reciprocals. Thus,

$$
\sqrt{\pi} \sigma_{1}\left(\frac{\pi}{6}\right)=-\frac{2}{3} \mathrm{~K}^{2}\left(\sin \frac{\pi}{12}\right)+\frac{2}{3} \pi,
$$

and

$$
\sqrt{\pi} \sigma_{2}\left(\frac{\pi}{6}\right)=\frac{1}{9} \mathrm{~K}^{2}\left(\sin \frac{\pi}{12}\right)+\frac{\pi^{2}}{18} \mathrm{~K}^{-2}\left(\sin \frac{\pi}{12}\right) .
$$

In consequence of Theorem 5 below, all $C_{3 \text {,even }}$ are superpositions of $C_{3,0}$ and $C_{3,2}$ with polynomial (in $k$ ) weights; thus, the $C_{3, \text { even }}$ can only involve algebraic combinations of the numbers above, such as $\log 2, \pi$ and the elliptic evaluations/integrals. PSLQ suggests no relations exist between the seven monomials implicit in (24).

\subsubsection{The $C_{3, \text { odd }}$ integrals}

A first observation on the cases $C_{k, \text { odd }}$ is as follows. We recall the exact $L$ function evaluation given in [8].:

$$
C_{3}:=C_{3,1}=\mathrm{L}_{-3}(2):=\sum_{m \geq 0}\left(\frac{1}{(3 m+1)^{2}}-\frac{1}{(3 m+2)^{2}}\right) .
$$


This knowledge on $C_{3,1}$ leads, via (16), to the remarkable $L$-function identity

$$
\mathrm{L}_{-3}(2)=\frac{2}{3} \sum_{h=0}^{\infty} \frac{1}{h+1} \frac{1}{\left(\begin{array}{c}
2 h+1 \\
h
\end{array}\right)}\left(1-\frac{1}{2}+\frac{1}{3}-\cdots+\frac{1}{2 h+1}\right)
$$

Observe that, via relation (12), this resolves the relevant Meijer- $G$ in terms of an $L$-function, which Meijer- $G$ identity we believe to be new.

Now, the $C_{3, \text { odd }}$ seem to be pairwise rationally related, in the following sense. We discovered via numerical experiments the conjectures ${ }^{2}$

$$
\begin{gathered}
C_{3,3} \stackrel{?}{=}-\frac{4}{27}+\frac{2}{9} \mathrm{~L}_{-3}(2), \\
C_{3,5} \stackrel{?}{=}-\frac{92}{1215}+\frac{8}{81} \mathrm{~L}_{-3}(2),
\end{gathered}
$$

and several more, suggesting rational relations $a C_{3, k}+b C_{3, k^{\prime}}=c$ for any distinct odd pair $\left(k, k^{\prime}\right)$, with $a, b, c$ rational, $a \neq b$. These $(n=3$, odd $k)$ conjectures were subsequently proven, as below. We should mention that we found no such rational relations whatever between pairs of $C_{3 \text {,even }}$ (see Conjecture 3 ).

One might conceivably use the residue expansion (16) to prove our experimentally detected relations. However there is another route, one that leads to an efficient algorithm for resolving the closed form of any $C_{3 \text {,odd }}$. We harken back to the dimensional-reduction methods in [8] and reduce to a 2-dimensional integral

$$
C_{3, k}=\frac{\sqrt{\pi}}{3 !} \frac{\Gamma\left(\frac{k+1}{2}\right)}{\Gamma\left(\frac{k}{2}+1\right)} \int_{0}^{\infty} \int_{0}^{\infty} \frac{d x d y}{x y} \frac{1}{\{(1+x+y)(1+1 / x+1 / y)\}^{(k+1) / 2}} .
$$

Now for odd $k$ we may assign $m:=(k-1) / 2$ and write

$$
\begin{gathered}
\int_{0}^{\infty} \int_{0}^{\infty} \frac{d x d y}{x y} \frac{1}{\{(1+x+y)(1+1 / x+1 / y)\}^{(k+1) / 2}}= \\
\left.\frac{1}{m !^{2}}\left(\frac{\partial}{\partial \alpha} \frac{\partial}{\partial \beta}\right)^{m} \int_{0}^{\infty} \int_{0}^{\infty} \frac{d x d y}{x y} \frac{1}{(\alpha+x+y)(\beta+1 / x+1 / y)}\right|_{\alpha, \beta=1}
\end{gathered}
$$

The integral over $x$, say, may then be done, after which we put $y=z / \beta$ to reveal that, remarkably, the $\alpha, \beta$-dependent integral is really a function only of the product $c:=\alpha \beta$. In fact,

$$
\begin{gathered}
\int_{0}^{\infty} \int_{0}^{\infty} \frac{d x d y}{x y} \frac{1}{(\alpha+x+y)(\beta+1 / x+1 / y)}=\int_{0}^{\infty} \frac{\log (1+1 / z)+\log (c+z)}{z^{2}+c z+c} d z \\
=\int_{0}^{1} \frac{\log c-2 \log t}{t^{2}-c t+c} d t
\end{gathered}
$$

\footnotetext{
${ }^{2}$ The notation $\stackrel{?}{=}$ means we experimentally suspect a given equality in absence of rigorous proof. Of course, we shall prove these $C_{3 \text {,odd }}$ closed forms, but we prefer to use $\stackrel{?}{=}$ when reporting on initial numerical discovery.
} 
the final integral being obtained by making the substitutions $1+1 / z=1 / t$ and $c+z=c / t$ respectively in the two parts of the preceding integral. Thus $C_{3, k}$ reduces to

$$
C_{3, k}=\left.\frac{2^{k+1}}{3 ! k !}\left(\frac{\partial}{\partial \alpha} \frac{\partial}{\partial \beta}\right)^{m} \Upsilon(\alpha \beta)\right|_{\alpha, \beta=1}
$$

where

$$
\begin{aligned}
\Upsilon(c) & :=\int_{0}^{1} \frac{\log \sqrt{c}-\log t}{t^{2}-c t+c} d t \\
& =\frac{1}{r_{+}-r_{-}}\left(-\frac{1}{2} \log \left(r_{+} r_{-}\right) \log \frac{1-1 / r_{-}}{1-1 / r_{+}}+\operatorname{Li}_{2}\left(1 / r_{-}\right)-\operatorname{Li}_{2}\left(1 / r_{+}\right)\right)
\end{aligned}
$$

with

$$
r_{ \pm}:=\frac{c \pm \sqrt{c^{2}-4 c}}{2} .
$$

Sure enough, for $k=1$, and so $m=0$ and no differentiation in (25), we obtain our original case $C_{3}:=C_{3,1}=(2 / 3) \Upsilon(1)=\mathrm{L}_{-3}(2)$.

More generally, our finite representation (25) leads to a proof of the evaluations above for $C_{3,3}$ and $C_{3,5}$ and indeed to a proof of our rational-relation conjecture. To this end, note that we can use the operator identity

$$
\frac{\partial^{2}}{\partial \alpha \partial \beta}=\frac{\partial}{\partial c} c \frac{\partial}{\partial c}
$$

valid on functions $f$ where $c=\alpha \beta$. In expanded form this means

$$
\left(\frac{\partial}{\partial \alpha} \frac{\partial}{\partial \beta}\right)^{m} f(c)=\sum_{k=0}^{m}\left(\begin{array}{c}
m \\
k
\end{array}\right) \frac{m !}{k !} c^{k} f^{(m+k)}(c) .
$$

From the above relations one may now derive, for nonnegative integers $m$,

$$
C_{3,2 m+1}=\frac{2^{2 m+1}}{3(2 m+1)\left(\begin{array}{c}
2 m \\
m
\end{array}\right)} \sum_{k=0}^{m}\left(\begin{array}{c}
m \\
k
\end{array}\right)\left(\begin{array}{c}
m+k \\
k
\end{array}\right)(-1)^{m+k+1} I(m+k),
$$

where

$$
I(\nu):=\int_{0}^{1} \frac{t^{\nu} \log t}{\left(t^{2}-t+1\right)^{\nu+1}} d t .
$$

These observations lead us to

Theorem 1 For odd $k \geq 1$, we have

$$
C_{3, k}=p_{3, k}+q_{3, k} \mathrm{~L}_{-3}(2),
$$

with the $p, q$ coefficients always being rational, $q_{3, k}$ being given explicitly by (30) below. 
Proof. In terms of the $I$ function in (27), establishing the recursion

$$
\nu I(\nu-1)+(2 \nu+1) I(\nu)-3(\nu+1) I(\nu+1)+\frac{1}{\nu}=0
$$

is enough to prove the theorem, because

$$
I(0)=-\frac{3}{2} \mathrm{~L}_{-3}(2), \quad I(1)=-\frac{1}{2} \mathrm{~L}_{-3}(2) .
$$

One may also derive

$$
I(\nu)=a_{\nu}+b_{\nu} \mathrm{L}_{-3}(2)
$$

with rational $a_{\nu}, b_{\nu}$ satisfying the recursions

$$
\begin{gathered}
\nu a_{\nu-1}+(2 \nu+1) a_{\nu}-3(\nu+1) a_{\nu+1}+\frac{1}{\nu}=0, \quad a_{0}=a_{1}=0 \\
\nu b_{\nu-1}+(2 \nu+1) b_{\nu}-3(\nu+1) b_{\nu+1}+\frac{1}{\nu}=0, \quad b_{0}=-\frac{3}{2}, b_{1}=-\frac{1}{2} .
\end{gathered}
$$

So we now prove the recursion $(28)$. For $x \in(-1,1)$ we have

$$
y(x):=\sum_{\nu=0}^{\infty} I(\nu) x^{\nu}=\int_{0}^{1} \frac{\log t}{t^{2}-t(1+x)+1} d t .
$$

The recursion (28) thus holds if and only if

$$
(x+1) \sum_{\nu=0}^{\infty} I(\nu) x^{\nu}+\left(x+2-\frac{3}{x}\right) \sum_{\nu=0}^{\infty} \nu I(\nu) x^{\nu}=I(0)-3 I(1)+\log (1-x)
$$

which is equivalent to $y$ satisfying the differential equation

$$
(x+1) y+\left(x^{2}+2 x-3\right) y^{\prime}=\log (1-x)-3 \mathrm{~L}_{-3}(2),
$$

subject to the initial condition

$$
y(0)=-\frac{3}{2} \mathrm{~L}_{-3}(2) .
$$

Maple verifies that $y(x)$ is indeed a solution.

QED

It turns out to be possible to give a finite expression for the $q_{3, k}$ rational in Theorem 1 . What may be called the terminal term of the chain differentiation in (25), namely

$$
\left.\left\{\operatorname{Li}_{2}\left(\frac{1}{r}{ }_{-}\right)-\operatorname{Li}_{2}\left(\frac{1}{r_{+}}\right)\right\} \cdot\left(\frac{\partial}{\partial \alpha} \frac{\partial}{\partial \beta}\right)^{m} \frac{1}{r_{+}-r_{-}}\right|_{\alpha, \beta=1},
$$

gives the rational coefficient of $\mathrm{L}_{-3}(2)$ as

$$
q_{3, k}=\left.\sqrt{3} \frac{2^{k-1}}{k !}\left(\frac{\partial}{\partial \alpha} \frac{\partial}{\partial \beta}\right)^{m} \frac{1}{(\alpha \beta(4-\alpha \beta))^{1 / 2}}\right|_{\alpha, \beta=1} .
$$


In particular, a finite expression for the general $q$ coefficient is, with $m:=$ $(k-1) / 2$,

$$
\begin{aligned}
q_{3, k} & =\frac{2^{k-1}}{k !} \sum_{j=0}^{m}\left(\begin{array}{c}
m \\
j
\end{array}\right)(-1)^{m+j} \frac{m !}{j !} \sum_{i=0}^{m+j}\left(\begin{array}{c}
m+j \\
i
\end{array}\right)\left(\frac{1}{2}\right)_{i}\left(\frac{1}{2}\right)_{m+j-i}\left(-\frac{1}{3}\right)^{i} \\
& =\sqrt{3} \frac{2^{2 m-1} m !}{(2 m-1) !} \sum_{j=0}^{m} \frac{(-1)^{m+j}\left(\begin{array}{c}
m \\
j
\end{array}\right)}{j !}{ }_{2} \mathrm{~F}_{1}\left(\begin{array}{c}
\frac{1}{2}, \frac{1}{2} \\
\frac{1}{2}-m-j
\end{array} ; \frac{1}{4}\right) \prod_{i=0}^{j}\left(\frac{1}{2}+m+i\right) .
\end{aligned}
$$

The above analysis provides closed forms for the relevant Meijer $G$-functions. The method also provides an algorithm for exact evaluation of any $C_{3, \text { odd }}$ rather efficiently. ${ }^{3}$ One may arrive quickly at such instances as

$$
\begin{aligned}
C_{3,15}:= & \frac{1}{3 !} \int_{-\infty}^{\infty} \int_{-\infty}^{\infty} \int_{-\infty}^{\infty} \frac{d x d y d z}{(\cosh x+\cosh y+\cosh z)^{16}} \\
& =-\frac{11884272896}{837856594575}+\frac{4139008}{227988189} \mathrm{~L}_{-3}(2) .
\end{aligned}
$$

\subsection{Evaluations of $C_{4, k}$}

We begin with the first case of (13). Residue calculus - again we omit the intricacies - gives series such as

$$
\begin{aligned}
C_{4,0} & =\frac{1}{24} \sum_{h=0}\left(\frac{\Gamma^{4}(h+1 / 2)}{\Gamma^{4}(h+1)}\right)^{\prime \prime} \\
& =\frac{1}{3} \sum_{h=0}^{\infty} \frac{\Gamma^{4}(h+1 / 2)}{\Gamma^{4}(h+1)}\left(8\left(-\log 2+H_{2 h}^{(-1)}\right)^{2}+\zeta(2)-2 H_{2 h}^{(-2)}\right)
\end{aligned}
$$

where the double-derivative " is with respect to $h$, and the new sum is

$$
H_{\mu}^{(-2)}:=1-1 / 2^{2}+1 / 3^{2}-\cdots \pm 1 / \mu^{2}
$$

with $H_{0}^{(-2)}:=0$. However, just is with the $C_{3 \text {,even }}$ cases of the previous section, we know not a single closed form for $C_{4 \text {,even }}$ and again, we found experimentally that $C_{4, \text { odd }}$ are pairwise rationally related, meaning (see Table 1 for $C_{4}:=C_{4,1}$ ) that every $C_{4, \text { odd }}$ would be $p+q \zeta(3)$ for rational $p, q$.

\footnotetext{
${ }^{3}$ One may explicitly differentiate and simplify in (25), but a faster algorithm is to use the finite expression for $q_{3, k}$ given after Theorem 1, an extreme-precision evaluation of series (16), then a function such as Mathematica's Rationalize [ ] to resolve $p_{3, k}$. This amounts to an interesting, systematic use of extreme precision within a general algorithm.
} 
The finite-form evaluation of any $C_{4, \text { odd }}$ is achieved as follows. Define integrals

$$
U_{h}:=\frac{i \pi}{2} \int_{-\infty}^{\infty} \frac{\sinh \pi t}{\cosh ^{3} \pi t}\left(-\frac{1}{2}+i t\right)^{h} d t=(-1)^{h+1} h(h-1) \frac{\zeta(2-h)}{2 \pi} .
$$

This later identity actually holds, for any integer $h$, with $U_{1}:=1 /(2 \pi)$. Note that under the further constraint $h \geq 0$, the quantity $\pi U_{h}$ for $h \geq 0$ is rational, as follows from the fact of known evaluations of $\zeta(2-h)$.

The relevance of the $U_{h}$ is that a Meijer contour-integral as in (13) can be developed like so:

$$
\begin{aligned}
G & :=\frac{1}{2 \pi i} \int_{\mathcal{C}} \frac{\Gamma^{3}(-s) \Gamma^{3}((k+1) / 2+s)}{\Gamma(1+k / 2+s) \Gamma(1 / 2-s)} d s \\
& =\frac{i \pi}{2} \int_{-\infty}^{\infty} \frac{\sinh \pi t}{\cosh ^{3} \pi t} F\left(-\frac{1}{2}+i t\right) d t
\end{aligned}
$$

where

$$
F(s):=\frac{\Gamma^{3}((1+k) / 2+s) \Gamma(1 / 2+s)}{\Gamma^{3}(1+s) \Gamma(1+k / 2+s)} .
$$

Now the key is, if we write

$$
F(s)=f(s)+\phi(s)
$$

where we express $F(s)=\sum_{j} f_{j} s^{j}$ as a polynomial and an error term $\phi(s)=o(s)$, then we can resolve the original Meijer- $G$ by employing the $U_{h}$ identity on the monomials $f_{j} s^{j}$, and using residue calculus for the $\phi$ term, to write

$$
G=\sum_{j} f_{j} U_{j}+\frac{1}{2 \pi} \sum_{h=0}^{\infty} \phi^{\prime \prime}(h) .
$$

This analysis now leads to a proof of the experimentally discovered conjecture on rational relations for any pair of $C_{4, \text { odd }}$ :

Theorem 2 For odd $k \geq 1$, we have

$$
C_{4, k}=p_{4, k}+q_{4, k} \zeta(3),
$$

with the $p, q$ coefficients always being rational. In particular, a finite expression for the general $q$ coefficient is, with $m:=(k-1) / 2$,

$$
q_{4, k}=\frac{7}{12} \frac{(2 m) !^{3}}{k ! \cdot 64^{m} m !^{4}} \sum_{j=0}^{m} \frac{\left(\begin{array}{c}
m \\
j
\end{array}\right)^{4}}{\left(\begin{array}{c}
2 m \\
2 j
\end{array}\right)^{3}}
$$


Proof. For fixed odd $k$ the function $F$ is indeed polynomial plus a decay term, namely, set $m:=(k-1) / 2$ and write

$$
\begin{aligned}
F(s) & =\frac{(1+s)^{3}(2+s)^{3} \cdots(m+s)^{3}}{(s+1 / 2)(s+3 / 2) \cdots(s+m+1 / 2)} \\
& =\sum_{j=0}^{2 m-1} f_{j} s^{j}+\sum_{j=0}^{m} \frac{A_{j}}{s+j+1 / 2} .
\end{aligned}
$$

Here, the coefficients $\left(f_{j}\right)$ and $\left(A_{j}\right)$ are all rational, and can be calculated exactly, using polynomial remaindering and partial-fraction expansion, respectively. Thus the original Meijer $G$-function from (13) is given exactly by the result (32)

$$
G=\sum_{j=0}^{2 m-1} f_{j} U_{j}+\frac{1}{2 \pi} \sum_{j=0}^{m} A_{j} \zeta(2, j+1 / 2),
$$

where $\zeta(s, a):=\sum_{h \geq 0} 1 /(h+a)^{s}$ is the Hurwitz zeta-function.

Now, being as each $U_{j}$ here is (rational) $/ \pi$, each $\zeta(2, j+1 / 2)$ is (rational) + (rational) $\zeta(3)$, and each $C_{4, \text { odd }}$ is (rational) $\pi G$, the theorem follows. The explicit evaluation of $q_{4, k}$ arises from the natural partial-fraction evaluation of the $A_{j}$ terms and the accumulation of all normalizing factors.

QED

This result amounts to a closed-form resolution of the Meijer $G$-function in (13) for any odd $k$ in terms of $\zeta(3), \pi$, and rationals. Moreover,

$$
\begin{aligned}
\sum_{j=0}^{m} \frac{\left(\begin{array}{c}
m \\
j
\end{array}\right)^{4}}{\left(\begin{array}{c}
2 m \\
2 j
\end{array}\right)^{3}}={ }_{4} \mathrm{~F}_{3}\left(\begin{array}{c}
1 / 2,1 / 2,1 / 2,1 \\
-m+1 / 2,-m+1 / 2,-m+1 / 2
\end{array} ;-1\right) \\
-\frac{\left(\begin{array}{c}
m \\
m+1
\end{array}\right)^{3}}{\left(\begin{array}{c}
2 m \\
2 m+2
\end{array}\right)^{3}}{ }_{4} \mathrm{~F}_{3}\left(\begin{array}{c}
m+3 / 2, m+3 / 2, m+3 / 2,1 \\
3 / 2,3 / 2,3 / 2
\end{array} ;-1\right) .
\end{aligned}
$$

In this way, as for $n=3$, polynomial-remaindering and rational-arithmetic algorithms quickly yield exact evaluations such as

$$
\begin{gathered}
C_{4,15}:=\frac{1}{4 !} \int_{-\infty}^{\infty} \int_{-\infty}^{\infty} \int_{-\infty}^{\infty} \int_{-\infty}^{\infty} \frac{d w d x d y d z}{(\cosh w+\cosh x+\cosh y+\cosh z)^{16}} \\
=-\frac{1744313209}{578605547520000}+\frac{67697}{26990346240} \zeta(3) .
\end{gathered}
$$

In general the odd Meijer-G form for $n=4$ can be written explicitly as

$$
C_{4,2 k+1}=\frac{1}{(2 k+1) !} \frac{\pi^{2}}{24} \int_{-\infty}^{\infty} \frac{\sinh (\pi t)}{t \cosh ^{3}(\pi t)} \prod_{j=1}^{k} \frac{(t-i(j-1 / 2))^{3}}{t-i j} d t
$$


while the even form, and those for $n=3$, offers less purchase. In particular, integration by parts in (34) yields

$$
C_{4,1}=\frac{\pi^{2}}{12} \int_{0}^{\infty} \frac{\tanh (t) \operatorname{sech}^{2}(t)}{t} d t=\frac{\pi}{24} \int_{0}^{\infty} \frac{\tanh ^{2}(\pi t)}{t^{2}} d t .
$$

We next substitute the partial fraction expansion

$$
\frac{\tanh (\pi y)}{y}=\frac{4}{\pi} \sum_{n=0}^{\infty} \frac{2 y}{4 y^{2}+(2 n+1)^{2}}
$$

and expand, then interchange integration and summation to obtain from

$$
\int_{0}^{\infty} \frac{4 y^{2}}{\left(4 y^{2}+(2 n+1)^{2}\right)\left(4 y^{2}+(2 m+1)^{2}\right)} d y=\frac{\pi}{(2 n+1)(2 m+1)(2 n+2 m+2)}
$$

that

$$
C_{4,1}=\frac{2}{3} \sum_{n=0}^{\infty} \sum_{m=0}^{\infty} \frac{1}{(2 n+1)(2 m+1)(2 n+2 m+2)} .
$$

This double sum is a Tornheim double sum or a Witten $\zeta$-value, see [14], and equals

$$
\begin{aligned}
\int_{0}^{1} \frac{\operatorname{arctanh}^{2}(x)}{x} d x & =\int_{0}^{1} \frac{\log ^{2} \sqrt{\frac{1-x}{1+x}}}{x} d x=\frac{1}{2} \int_{0}^{1} \frac{\log ^{2} t}{1-t^{2}} d t \\
& =\sum_{n=1}^{\infty} \frac{1}{(2 n-1)^{3}}=\frac{7}{8} \zeta(3),
\end{aligned}
$$

where the first integral and penultimate sum are obtained on integrating termwise. Thus,

$$
C_{4,1}=\frac{7}{12} \zeta(3)
$$

as before. Similar machinations lead to a corresponding evaluation of $C_{4,3}$.

\section{Recursion relations-experiment}

Based on extensive computational work we conjecture that:

Conjecture 1 For given $n \in \mathbb{Z}^{+}$with $M:=\lfloor(n+1) / 2\rfloor$, The integrals $\left(C_{n, k}\right)$ enjoy an order- $M$ recursion involving $M+1$ terms with coefficients being integral polynomials $P_{n, j}$ each of degree $n$, that is

$$
P_{n, 0}(k) C_{n, k}+P_{n, 1}(k) C_{n, k+2}+\cdots+P_{n, M}(k) C_{n, k+2 M}=0 .
$$

Moreover, this holds for all complex $k$ in the sense of analytic continuation (the existence of poles in the $k$-plane is admitted). 
We shall eventually be able to prove certain instances of Conjecture 1; specifically, recursion relations amongst the $C_{n, k}$ with fixed $n=1,2,3,4$. The first open cases of Conjecture 1 are $n=5,6$, specifically:

$$
\begin{aligned}
0 \stackrel{?}{=} & (k+1)^{5} C_{5, k}-(k+2)\left(35 k^{4}+280 k^{3}+882 k^{2}+1288 k+731\right) C_{5, k+2} \\
& +(k+2)(k+3)(k+4)\left(259 k^{2}+1554 k+2435\right) C_{5, k+4} \\
& -225(k+2)(k+3)(k+4)(k+5)(k+6) C_{5, k+6}
\end{aligned}
$$

and

$$
\begin{aligned}
0 \stackrel{?}{=} & (k+1)^{6} C_{6, k}-8(k+2)^{2}\left(7 k^{4}+56 k^{3}+182 k^{2}+280 k+171\right) C_{6, k+2} \\
& +16(k+2)(k+3)^{2}(k+4)\left(49 k^{2}+294 k+500\right) C_{6, k+4} \\
& -2304(k+2)(k+3)(k+4)^{2}(k+5)(k+6) C_{6, k+6}
\end{aligned}
$$

where as before the question mark is used to emphasize the fact that we have no formal proof.

Note that, on this conjecture, our renormalized (lowercase-notated) $c_{n, k}=$ $\Gamma(k+1) n ! 2^{-n} C_{n, k}$ of equation (8) then satisfies a recursion with a straightforward polynomial adjustment:

$$
\sum_{i=0}^{M}(-1)^{i} p_{n, i}(k+i+1) c_{n, k+2 i}=0 .
$$

We write the "little- $c$ " recursion in this way for convenient connection with experimental results; for example, we have always encountered natural alternating signs, and some obvious factors of the polynomials $p$ implicitly defined by (37). Note, for instance, that the experimental recursions (35) and (36) can be recast compactly in the form of (37) by defining

$$
\begin{array}{ll}
p_{5,0}(x)=x^{6} & p_{6,0}(x)=x^{7} \\
p_{5,1}(x)=35 x^{4}+42 x^{2}+3 & p_{6,1}(x)=x\left(56 x^{4}+112 x^{2}+24\right) \\
p_{5,2}(x)=259 x^{2}+104 & p_{6,2}(x)=x\left(784 x^{2}+944\right) \\
p_{5,3}(x)=225 & p_{6,3}(x)=2304 x .
\end{array}
$$

Table 2 has many other $p_{n, i}$ polynomials that we have found experimentally.

There is actually a substantial literature on such recursions. Most authors abide by the nomenclature as we do, namely, the order of the recursion is $M$, meaning there are $M+1$ different $C$ terms (and $M+1$ polynomial coefficients). Some researchers refer to any sequence such as $C$, satisfying such a recursion, as holonomic, and observe that a generating function will satisfy a similar recurrence relation in its derivatives [37, 47, 20].

Two more conjectures also experimentally motivated are

Conjecture 2 Fix $n$ and a complex rational $k_{0}$. Then for $k$ lying in the arithmetic progression $\ldots, k_{0}-4, k_{0}-2, k_{0}, k_{0}+2, k_{0}+4, \ldots$, the set $\left(C_{n, k}: k \in\right.$ $\left.k_{0}+2 \mathbb{Z}\right)$ is rationally generated by any $M:=\lfloor(n+1) / 2\rfloor$ distinct elements, but no fewer. 
Conjecture 3 For distinct complex pair $\left(k, k^{\prime}\right)$ the rational relation $p C_{n, k}+$ $q C_{n, k^{\prime}}=r$ with $p, q, r$ complex rationals, $p \neq q$, is impossible for $n \geq 5$. For $n=3,4$ the rational relation is only possible for both $k, k^{\prime}$ odd integers.

Since all of these conjectures have been experimentally motivated, we hereby start our recursion discussion in the historical spirit, with experimental results first (and knowing that some of the tabulated recursions in the present section are proven and some are not). We give our substantial evidence in Table 2, where $c_{n, k}$ (lowercase notation) is defined in (8), and in Table 3.

An example of our experimental forays runs as follows. The form of the non-trivial coefficients for a possible recursion for the $C_{3, k}$ and $C_{4, k}$ was assisted by consulting Sloane's Online Encyclopedia ${ }^{4}$ which for $C_{4, k}$ connected the coefficients to the sequence A063495. ${ }^{5}$ Having found these recursions, it was then reasonable to assume the coefficients were polynomials of the conjectured degree; and the Tables were then built by numerical interpolation after the use of PSLQ. The predicted recursions were then numerically checked to extreme-precision at various values of $k$.

Table 2 shows recursions for the renormalized $c_{n, k}:=k ! n ! 2^{-n} C_{n, k}$, for $1 \leq n \leq 12$ and integer $k$. Using the recursion form (37) we end up with simple (odd or even, positive) polynomials $p_{n, i}$. The explicit polynomials $p_{n, i}$ that we have found experimentally are shown in Tables 2 and 3.

In particular we conjecture from Table 3 that

$$
\begin{aligned}
p_{n, 0}(x)= & x^{n+1} \\
p_{n, 1}(x)= & \sum_{j=1}^{M} j\left(\begin{array}{c}
n+2 \\
2 j+1
\end{array}\right) x^{n+1-2 j} \\
= & \frac{1}{4}(n+1+x)(x-1)^{n+1}+\frac{1}{4}(x+1)^{n+1}(n+1-x) \\
p_{n, 2}(x)= & \sum_{j=1}^{M-1} \frac{j 4^{j-1}((2 j+3)(n+2)+j+1}{j+2} \cdot\left(\begin{array}{c}
n+2 \\
2 j+3
\end{array}\right) x^{n-1-2 j} \\
= & \frac{1}{32}\left((n+x+2)^{2}-\frac{7 n}{2}-\frac{11(x+2)}{4}\right)(x-2)^{n+1} \\
& +\frac{1}{32}\left((n-x+2)^{2}-\frac{7 n}{2}+\frac{11(x-2)}{4}\right)(x+2)^{n+1} \\
& -\frac{1}{16} x^{n+1}\left(x^{2}-(n+2)^{2}\right) \\
& \cdots \\
p_{n, M}(x)= & \left\{\prod_{j=0}^{M}(n-2 j)^{2}\right\} x^{n-2 M} .
\end{aligned}
$$

${ }^{4}$ See www.research.att.com/ njas/sequences/index.html.

${ }^{5}$ Consult A063495 which makes reference to equation (10) in [24]. 


\begin{tabular}{|c|c|}
\hline$n$ & $i=1$ \\
\hline 1 & 1 \\
\hline 2 & $4 x$ \\
\hline 3 & $2+10 x^{2}$ \\
\hline 4 & $x\left(12+20 x^{2}\right)$ \\
\hline 5 & $3+42 x^{2}+35 x^{4}$ \\
\hline 6 & $x\left(24+112 x^{2}+56 x^{4}\right)$ \\
\hline 7 & $4+108 x^{2}+252 x^{4}+84 x^{6}$ \\
\hline 8 & $x\left(40+360 x^{2}+504 x^{4}+120 x^{6}\right)$ \\
\hline 9 & $5+220 x^{2}+990 x^{4}+924 x^{6}+165 x^{8}$ \\
\hline 10 & $x\left(60+880 x^{2}+2376 x^{4}+1584 x^{6}+220 x^{8}\right)$ \\
\hline 11 & $6+390 x^{2}+2860 x^{4}+5148 x^{6}+2574 x^{8}+286 x^{10}$ \\
\hline 12 & $x\left(84+1820 x^{2}+8008 x^{4}+10296 x^{6}+4004 x^{8}+364 x^{10}\right)$ \\
\hline$n$ & $i=2$ \\
\hline 3 & 9 \\
\hline 4 & $64 x$ \\
\hline 5 & $104+259 x^{2}$ \\
\hline 6 & $x\left(944+784 x^{2}\right)$ \\
\hline 7 & $816+4752 x^{2}+1974 x^{4}$ \\
\hline 8 & $x\left(9024+17520 x^{2}+4368 x^{4}\right)$ \\
\hline 9 & $5376+54384 x^{2}+52800 x^{4}+8778 x^{6}$ \\
\hline 10 & $x\left(70144+236544 x^{2}+137808 x^{4}+16368 x^{6}\right)$ \\
\hline 11 & $32000+492544 x^{2}+830544 x^{4}+322608 x^{6}+28743 x^{8}$ \\
\hline 12 & $x\left(481280+2469376 x^{2}+2498496 x^{4}+693264 x^{6}+48048 x^{8}\right)$ \\
\hline$n$ & $i=3$ \\
\hline 5 & 225 \\
\hline 6 & $2304 x$ \\
\hline 7 & $7796+12916 x^{2}$ \\
\hline 8 & $x\left(94976+52480 x^{2}\right)$ \\
\hline 9 & $170298+625196 x^{2}+172810 x^{4}$ \\
\hline 10 & $x\left(2409216+2949056 x^{2}+489280 x^{4}\right)$ \\
\hline 11 & $2999076+18232188 x^{2}+11161436 x^{4}+1234948 x^{6}$ \\
\hline 12 & $x\left(48354048+98000448 x^{2}+36003968 x^{4}+2846272 x^{6}\right)$ \\
\hline$n$ & $i=4$ \\
\hline 7 & 11025 \\
\hline 8 & $147456 x$ \\
\hline 9 & $851976+1057221 x^{2}$ \\
\hline 10 & $x\left(13036544+5395456 x^{2}\right)$ \\
\hline 11 & $39605040+106102880 x^{2}+21967231 x^{4}$ \\
\hline 12 & $x\left(683253760+610355200 x^{2}+75851776 x^{4}\right)$ \\
\hline$n$ & $i=5$ \\
\hline 9 & 893025 \\
\hline 10 & $14745600 x$ \\
\hline 11 & $129879846+128816766 x^{2}$ \\
\hline 12 & $x\left(2393358336+791691264 x^{2}\right)$ \\
\hline$n$ & $i=6$ \\
\hline 11 & 108056025 \\
\hline 12 & $2123366400 x$ \\
\hline
\end{tabular}

Table 2: Experimental polynomials $p_{n, i}$ for $1 \leq i \leq 6$ and $1 \leq n \leq 12$. 


\begin{tabular}{|c|c|c|c|c|}
\hline$n$ & $i=0$ & $i=1$ & $i=2$ & $i=3$ \\
\hline 1 & $x^{2}$ & $\mathbf{1}$ & & \\
2 & $x^{3}$ & $\mathbf{4} x$ & 9 & \\
\hline 3 & $x^{4}$ & $\overline{2}+\mathbf{1 0} x^{2}$ & $64 x$ & \\
4 & $x^{5}$ & $x\left(\overline{12}+\mathbf{2 0} x^{2}\right)$ & $104+259 x^{2}$ & 225 \\
\hline 5 & $x^{6}$ & $3+\overline{42} x^{2}+\mathbf{3 5} x^{4}$ & $x\left(944+784 x^{2}\right)$ & $2304 x$ \\
6 & $x^{7}$ & $x\left(24+\overline{112} x^{2}+\mathbf{5 6} x^{4}\right)$ & $816+4752 x^{2}+1974 x^{4}$ & $7796+12916 x^{2}$ \\
\hline 7 & $x^{8}$ & $4+108 x^{2}+\overline{252} x^{4}+\mathbf{8 4 4} x^{6}$ & & \\
8 & $x^{9}$ & $x\left(40+360 x^{2}+\overline{504} x^{4}+\mathbf{1 2 0} x^{6}\right)$ & $x\left(9024+17520 x^{2}+4368 x^{4}\right)$ & $x\left(94976+52480 x^{2}\right)$ \\
\hline
\end{tabular}

Table 3: Polynomials $p_{n, i}$ for $0 \leq i \leq 3$ and $1 \leq n \leq 8$. Note the coefficient of the rightmost polynomial is $(1 \cdot 3 \cdots \cdots n)^{2}$ or $(2 \cdot 4 \cdots \cdots n)^{2}$ respectively. Correspondingly, the bold numbers are of the form $\left(\begin{array}{l}n \\ 3\end{array}\right)$, while the overlined numbers are of the form $2\left(\begin{array}{l}n \\ 5\end{array}\right)$, etc. Generally, MacMahon's numbers, see Sloane's A008955, seem closely related: $T(n, k)=T(n, k-1)+k^{2} T(n-1, k-1)$.

Recall that $M:=\lfloor(n+1) / 2\rfloor$ is the recursion order and we set $p_{n, i}=0$ for $i \geq M$. If we consider the graded generating function we equivalently conjecture that

$$
G_{i}(x, y):=\sum_{n=1}^{\infty} p_{n, i}(x) y^{n}
$$

we obtain

$$
\begin{aligned}
G_{0}(x, y) & =\frac{x}{1-x y} \\
G_{1}(x, y) & =\frac{1}{(x y+y-1)^{2}(x y-y-1)^{2}} \\
G_{2}(x, y) & =\frac{y^{3}\left(-(1-x y)^{3}+10(1-x y)^{2}+4 y^{2}(1-x y)-8 y^{2}\right)}{(x y+2 y-1)^{3}(1-x y)^{3}(x y-2 y-1)^{3}},
\end{aligned}
$$

but have no idea what the general pattern should be.

\section{Recursion relations-theory}

\subsection{Direct methods}

An immediate but demonstrative result that does not require experimental mathematics is

Theorem 3 Conjecture 1 is true for $n=1,2$. In fact, for any complex $k$,

$$
(k+1) C_{1, k}-(k+2) C_{1, k+2}=0
$$

and

$$
(k+1)^{2} C_{2, k}-4(k+2)^{2} C_{2, k+2}=0 .
$$


Proof. The desired recursions follow immediately and analytically from (14) and (15) respectively.

QED

As intimated in Section 4, PSLQ in tandem with Sloane suggests that the $C_{3, k}$ satisfy a definite recursion, at least for integers $k$. We can get a foothold on this, with a view to the general analytic Conjecture 1, with

Theorem 4 Set $n=3$, whence for positive odd integers $k$ we have

$$
\begin{aligned}
0 & =(k+1)^{3} C_{3, k}-2(k+2)\left(5(k+2)^{2}+1\right) C_{3, k+2} \\
& +9(k+2)(k+3)(k+4) C_{3, k+4} .
\end{aligned}
$$

Remark. We shall eventually prove the recursion for general complex $k$; however, the two "direct" methods of proof here for odd $k$ are instructive and have, indeed, led us into the more general analytical forays to follow.

Proof (first method). For nonnegative integer $m$, we begin with the formulae for $C_{3,2 m+1}$ and $I(\nu)$, namely $(26,27)$ respectively. We now make the crucial observation that

$$
\sum_{k=0}^{n} \frac{\left(\begin{array}{c}
n \\
k
\end{array}\right)\left(\begin{array}{c}
n+k \\
k
\end{array}\right) t^{n+k} \log t}{\left(t-t^{2}-1\right)^{n+k+1}}=P_{n}\left(1-\frac{2 t}{-t+t^{2}+1}\right) \frac{t^{n} \log t}{\left(t-t^{2}-1\right)^{n+1}},
$$

and so

$$
C_{3,2 m+1}=\frac{1}{3} \frac{2^{2 m+1}}{(2 m+1)\left(\begin{array}{c}
2 m \\
m
\end{array}\right)} \int_{0}^{1} P_{m}\left(1-\frac{2 t}{t^{2}-t+1}\right) \frac{t^{m} \log t}{\left(t-t^{2}-1\right)^{m+1}} d t
$$

where $P_{n}$ is the $n$-th Legendre polynomial with ordinary generating function, see [1],

$$
\sum_{n=0}^{\infty} P_{n}(x) y^{n}=\frac{1}{\sqrt{1-2 x y+y^{2}}}
$$

Let $J_{m}$ denote the integral on the right-hand side of (45). From (45) and (46), on justifying the exchange of sum and integral, we obtain that the generating function for $J_{m}$ is

$$
J(x):=\sum_{\mu \geq 0} J_{\mu} x^{\mu}=\int_{0}^{1} \frac{\log t}{\sqrt{\left(-t+t^{2}+1+t x\right)^{2}-4 t^{2} x}} d t .
$$

Now, our hypothesized recursion, when written for $J_{m}$ is

$$
m^{2} J_{m-1}-\left(3+10 m^{2}+10 m\right) J_{m}+9(m+1)^{2} J_{m+1}=0
$$

Thus it suffices to show that $J=v$ satisfies the ODE

$$
(x-3) v+\left(3 x^{2}-20 x+9\right) v^{\prime}+\left(x^{3}-10 x^{2}+9 x\right) v^{\prime \prime}=3 .
$$


This is indeed the case. Maple easily confirms that the value of the left-hand side of (48) is 3 .

QED

Proof (second method). Alternatively we observe that (26) writes $C_{3,2 m+1}=$ $a_{m} J_{m}$ where $a(0)=2 / 3$ and

$$
(-2 m-2) a(m)+(2 m+3) a(m+1)=0,
$$

while $J_{m}$ satisfies (47) — or via the proven recursion

$$
(n+1)^{2} u(n)+(n+1)(2 n+3) u(n+1)-3(n+2)(n+1) u(n+2)=-1,
$$

for $I$. The INRIA-designed Maple package 'gfun' provides an algorithm which will then produce a recursion for $C_{3,2 n+1}$ which simplifies to the vanishing of

$4(m-1)^{3} J_{m-2}-2(2 m-1)\left(3+10 m^{2}-10 m\right) J_{m-1}+9(2 m+1)(2 m-1) m J_{n}$

which Maple easily confirms to be as claimed. This proof also can be obtained in Mathematica using Carsten Schneider's 'Sigma' package available from RiscLinz, [33]. Both programs can certify the result, for example in Mathematica using 'CreativeTelescoping'.

QED

The coefficients $J_{m}$ are interesting in their own right. In fact,

$$
J_{m}=q_{m} L_{-3}(2)-p_{m} \rightarrow_{m} 0
$$

where for $m \geq 1$

$$
q_{m}=\frac{1}{2}-\sum_{k=1}^{m-1} 9^{-k} \mathrm{~F}_{2}\left(\begin{array}{c}
\frac{1}{2},-k,-k \\
1,2
\end{array} 4\right) .
$$

The first 6 values of $p_{m}$ and $q_{m}$ respectively are

$$
\left(p_{0}, \ldots, p_{5}\right)=\frac{1}{3}, \frac{23}{108}, \frac{145}{972}, \frac{1331}{11664}, \frac{242353}{2624400}, \frac{5495507}{70858800},
$$

and

$$
\left(q_{0}, \ldots, q_{5}\right)=\frac{1}{2}, \frac{5}{18}, \frac{31}{162}, \frac{71}{486}, \frac{517}{4374}, \frac{11723}{118098} .
$$

\subsection{Analytic method}

Presumably there are direct methods, analogous to those used for Theorem 4 , that would establish the experimentally motivated recursion for the $C_{4, \text { odd }}$. However, it turns out that an analytic approach handles both $C_{3, k}$ and $C_{4, k}$ recursions and moreover, does this for general complex $k$. Incidentally, by "general complex $k$ " here and elsewhere, we mean either that $C_{n, k}$ is defined as its original integral (1) and all $k \in \mathcal{K}$ are being considered, or we are contemplating the analytic continuation $C_{n, k}$ over the entire complex $k$-plane (and at poles recursions still make divergent sense).

The following method of proof, relying on a contour-integral application of the Zeilberger algorithm [39, 12, 48], was suggested to us by W. Zudilin [50]. 
Theorem 5 The recursion in Theorem 4 for $C_{3, \text { odd } k}$ extends to complex $k$; moreover, there is a recursion of the same order $(M=2)$ for the $C_{4, k}$. Explicitly, both of the recursions

$$
\begin{array}{r}
(k+1)^{3} C_{3, k}-2(k+2)\left(5(k+2)^{2}+1\right) C_{3, k+2}+9(k+2)(k+3)(k+4) C_{3, k+4} \\
=0 \\
(k+1)^{4} C_{4, k}-4(k+2)^{2}\left(5(k+2)^{2}+3\right) C_{4, k+2}+64(k+2)(k+3)^{2}(k+4) C_{4, k+4} \\
=0
\end{array}
$$

hold for general complex $k$.

Proof. (i) We focus on the $n=4$ case - the $n=3$ case follows the same logicusing a representation based on the Meijer form (13) and its associated contour integral. Contemplating $t$ as a complex variable, we have

$$
C_{4,2 t-1}=-\frac{\pi^{2}}{24 \pi i} \int_{-1 / 2-i \infty}^{-1 / 2+i \infty} F_{4}(t, s) \frac{\cos \pi s}{\sin ^{3} \pi s} d s,
$$

with the definition

$$
F_{4}(t, s):=\frac{\Gamma(s+1 / 2) \Gamma(s+t)^{3}}{\Gamma(2 t) \Gamma(s+1)^{3} \Gamma(1 / 2+s+t)} .
$$

If one then employs the Zeilberger algorithm ${ }^{6}$ one finds that the definition

$G_{4}(t, s):=s^{3} \frac{12 t^{3}+16 t-2+26 s t^{2}-26 t^{2}-37 t s+11 s+18 s^{2} t+4 s^{3}-12 s^{2}}{(t-1)(2 s+2 t-1)} F_{4}(t, s)$,

leads to

$$
\begin{gathered}
16 t^{2}(2 t+1)(2 t-1) F_{4}(t+1, s)-(2 t-1)^{2}\left(5 t^{2}-5 t+2\right) F_{4}(t, s) \\
+(t-1)^{4} F_{4}(t-1, s)=G_{4}(t, s+1)-G_{4}(t, s) .
\end{gathered}
$$

Inserting this $F, G$ relation into the contour integral yields

$$
\begin{aligned}
& 16 t^{2}(2 t+1)(2 t-1) C_{4,2 t+1}-(2 t-1)^{2}\left(5 t^{2}-5 t+2\right) C_{4,2 t-1}+(t-1)^{4} C_{4,2 t-3} \\
= & \frac{\pi^{2}}{24 \pi i} \int_{\mathcal{C}} G_{4}(t, s) \frac{\cos \pi s}{\sin ^{3} \pi s} d s,
\end{aligned}
$$

where now the contour $\mathcal{C}$ is an infinitely tall, thin rectangle running vertically through $-1 / 2+0 i$ and $1 / 2+0 i$.

However, this rectangular integral is zero, since the only singularity is at $s=0$, and as we saw in our previous Meijer analysis for $C_{4, k}$, the residue contribution is proportional to $\partial^{2} G_{4}(t, s) /\left.\partial s^{2}\right|_{s=0}$, which is zero. Thus, the recursion (49) holds in an analytic sense, and upon $t \rightarrow(k+3) / 2$ becomes the order-2 recursion desired.

\footnotetext{
${ }^{6}$ Say, by calling in Maple zeil (F4 $\left.(\mathrm{t}-1, \mathrm{~s}), \mathrm{s}, \mathrm{t}, \mathrm{N}, 2\right)$.
} 
(ii) For $n=3$, the same procedure goes through; we first harken back to Meijer representation (12) then define

$$
F_{3}(t, s):=\frac{\Gamma(s+1 / 2) \Gamma(s+t)^{2}}{\Gamma(2 t) \Gamma(s+1)^{3}},
$$

then run the Zeilberger algorithm to achieve

$$
G_{3}(t, s):=s^{3} \frac{12 t^{3}-17 t^{2}+14 s t^{2}-10 s t+6 t+4 s^{2} t-s^{2}+2 s-1}{2 t(t-1)} F_{3}(t, s)
$$

and

$$
\begin{gathered}
(4 t+1)(2 t+1)(2 t-1)(4 t-1) F_{3}(t+1, s)-t(2 t-1)\left(10 t^{2}-10 t+3\right) F_{3}(t, s) \\
+t(t-1)^{3} F_{3}(t-1, s)=G_{3}(t, s+1)-G_{3}(t, s) .
\end{gathered}
$$

Then, as with the $n=4$ case above, we observe the vanishing of the relevant contour integral and arrive at the correct recursion involving $C_{3,2 t-1}$. QED

\section{Continued fractions}

It will have occurred to many readers that the order $M=2$ recurrences, namely for the $C_{3, k}$ and $C_{4, k}$, should give rise to continued fractions, being as such fractions are also governed by order-2 recurrences. The classical Pincherle theorem [23, Theorem 7, p.202], [19] runs like so:

Theorem 6 (Pincherle) Let $\left(a_{N}: N \in \mathbb{Z}^{+}\right),\left(b_{N}: N \in \mathbb{Z}^{+}\right),\left(G_{N}: N=\right.$ $-1,0,1,2, \ldots)$ be sequences of complex numbers related for all $N \in \mathbb{Z}^{+}$by

$$
G_{N}=b_{N} G_{N-1}+a_{N} G_{N-2},
$$

with each $a_{N} \neq 0$. Denote by $P_{N} / Q_{N}$ the convergents to the continued fraction

$$
x:=\frac{a_{1}}{b_{1}+\frac{a_{2}}{b_{2}+\ldots}} .
$$

If $\lim _{N} G_{N} / Q_{N}=0$ then the fraction converges and has the value

$$
x=-\frac{G_{0}}{G_{-1}} .
$$

The Pincherle theorem may be applied to recursions of the form in Conjecture 1 when $n=3$ or 4 , as established in Theorem 5 . For these $n$ we have order- 2 recursions:

$$
P_{n, 0}(k) C_{n, k}+P_{n, 1}(k) C_{n, k+2}+P_{n, 2}(k) C_{n, k+4}=0 .
$$


If we identify $G_{N}:=C_{n, 2 N+2}$ the Pincherle theorem applies with

$$
\begin{aligned}
& b_{N}:=-\frac{P_{n, 1}(2 N-2)}{P_{n, 2}(2 N-2)}, \\
& a_{N}:=-\frac{P_{n, 0}(2 N-2)}{P_{n, 2}(2 N-2)},
\end{aligned}
$$

and we obtain a continued fraction with value $x=-C_{n, 2} / C_{n, 0}$. Similarly, setting $G_{N}:=C_{n, 2 N+3}$ and suitably modifying the definitions of $a_{N}, b_{N}$ gives us a fraction with value $-C_{n, 3} / C_{n, 1}$.

These machinations result in at least four attractive continued fractions having integer elements. Even though we do not know a single individual value of

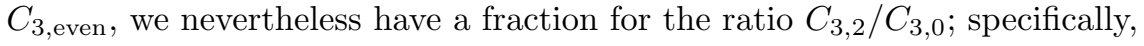

$$
18 \frac{C_{3,2}}{C_{3,0}}=\frac{9 \cdot 1^{4}}{d(1)-\frac{9 \cdot 3^{4}}{\ddots-\frac{9 \cdot(2 N-1)^{4}}{d(N)-\ldots}}}
$$

where $d(N):=40 N^{2}+2$. The very form of the fraction elements suggests that this ratio could well be a rational multiple of some brand of $L$-function, but we have not extensively searched for such.

For the $L$-function that appears in $C_{3 \text {,odd }}$ evaluations, we obtain

$$
\frac{2}{\mathrm{~L}_{-3}(2)}=3-\frac{9 \cdot 1^{4}}{f(1)-\frac{9 \cdot 2^{4}}{\ddots-\frac{9 \cdot N^{4}}{f(N)-\ldots}}}
$$

with $f(N):=10 N^{2}+10 N+3$, and so $f(0)=3$.

Along the same lines one derives a fraction

$$
16 \frac{C_{4,2}}{C_{4,0}}=\frac{1^{6}}{e(1)-\frac{3^{6}}{\ddots-\frac{(2 N-1)^{6}}{e(N)-\ldots}}}
$$

where $e(N):=N\left(20 N^{2}+3\right)$.

Finally, for the $C_{4, \text { odd }}$ we arrive at a fraction for $\zeta(3)$ :

$$
\frac{12}{7 \zeta(3)}=2-\frac{16 \cdot 1^{6}}{g(1)-\frac{16 \cdot 2^{6}}{\ddots-\frac{16 \cdot N^{6}}{g(N)-\cdots}}},
$$


where $g(N):=(2 N+1)\left(5 N^{2}+5 N+2\right)$, and so $g(0)=2$. This fraction is structurally reminiscent of the Apéry continued fraction for $\zeta(3)$. (See [17] and the references therein.) However, the arguments presented in [44] — where are derived Catalan-constant and $\zeta(4)$ fractions structurally similar to our $L$ and $\zeta(3)$ fractions above - suggest that irrationality proofs using such fractions are rare. Typically, certain number-theoretical properties of a recursion must be satisfied for an irrationality proof to be achievable.

Indeed there are many literature connections involving recursions, continued fractions, and irrationality [5, 26, 45, 31, 38, 46, 48]. Indeed, our recursion for $C_{4, k}$ in Theorem 5 (essentially a recursion relevant to $\zeta(3)$ ) can be found in the literature [4, p.23], and another one for the $C_{3, k}$, and so relevant to $L_{-3}(2)$ can be found also [49]. We note that irrationality proofs of Apéry type do not appear to arise from the recursions of the present paper. Indeed, to our knowledge the number $L_{-3}(2)$ has never been proven irrational.

\section{Further analytic properties of the $C_{n, k}$}

We have investigated interindicial relations of $k$-variant form, i.e. recursion relations, but now we turn to relations where the first index, $n$, varies.

\subsection{Analytic convolution}

On another idea of $\mathrm{W}$. Zudilin [50], we sought relations on the first index, namely the $n$ of $C_{n, k}$. One result is an analytic convolution theorem, where we recall the definition of the half-plane $\mathcal{K}$ from Section 1 , and the renormalization $c_{n, k}:=\Gamma(k+1) 2^{-n} n ! C_{n, k}$ :

Theorem 7 For complex $k \in \mathcal{K}$, positive integer $n$, and integer $q \in[1, n-1]$ we have

$$
c_{n, k}=\frac{1}{2 \pi i} \int_{\mathcal{C}} c_{n-q, k+s} c_{q,-1-s} d s,
$$

where the contour $\mathcal{C}$ runs vertically over $(\lambda-i \infty, \lambda+i \infty)$ with $\Re(\lambda) \in(-1,0)$.

Remark. There are at least two remarkable features of this result. First, this is a kind of recursion on the first index of the $c_{n, k}$ in contrast with the $k$-recursions; and second, the convolution surprisingly takes the same form for any (legal) indicial offset $q$.

Proof. Write our original definition (1) in the form

$C_{n, k}:=\frac{1}{n !} \int d x_{1} \cdots d x_{n}(A+B)^{-k-1}=\frac{1}{n !} \int d x_{1} \cdots d x_{n} A^{-k-1}(1+B / A)^{-k-1}$

where $A$ is the sum of the first $(n-q) \cosh$ terms, and $B$ is the sum of the remaining $q$ cosh terms. We then invoke the hypergeometric form of the binomial 
theorem, namely

$$
(1+B / A)^{-k-1}=\frac{1}{\Gamma(k+1)} \frac{1}{2 \pi i} \int_{\mathcal{C}} \Gamma(1+k+s) \Gamma(-s)(B / A)^{s} d s .
$$

We can then contemplate integration of $A$ terms over $d x_{1} \cdots d x_{n-q}$, and $B$ terms over $d x_{n-q+1} \cdots d x_{n}$, to obtain

$$
C_{n, k}=\frac{1}{\left(\begin{array}{c}
n \\
q
\end{array}\right)} \frac{1}{\Gamma(k+1)} \frac{1}{2 \pi i} \int_{\mathcal{C}} \Gamma(k+1+s) \Gamma(-s) C_{n-q, k+s} C_{q,-1-s} d s .
$$

But upon renormalization to the little-c forms, this is the statement of the theorem.

QED

We have not explored all of the implications of this theorem. However, we can use it to extend the reach, if you will, of Meijer- $G$ analysis. Though we encountered in Section 2 a certain blockade at $n=5$-namely we "ran out" of Meijer representations - we can nevertheless cast $C_{n, k}$ as an order- $\lfloor(n-1) / 2\rfloor$ nested-Barnes integral. Evidently, then, the Meijer representations (12, 13) can be considered in the larger scheme of things as the nested-Barnes cases for $n=3,4$.

The first nontrivial case of this "Meijer-Barnes extension" uses Theorem 7 with $n=5, q=2$ to yield

$$
\begin{aligned}
c_{5, k} & =\frac{1}{2 \pi i} \int_{\mathcal{C}_{s}} c_{2, k+s} c_{3,-1-s} d s \\
& =-\frac{1}{4 \pi^{2}} \int_{\mathcal{C}_{s}} \int_{\mathcal{C}_{t}} c_{2, k+s} c_{2,-1-s+t} c_{2,-1-t} d s,
\end{aligned}
$$

using the contours

$$
\mathcal{C}_{s}:=(\lambda-i \infty, \lambda+i \infty) \quad \text { and } \quad \mathcal{C}_{t}:=(\rho-i \infty, \rho+i \infty)
$$

where conditions simultaneously sufficient for these contours are:

$$
\Re(k)+\lambda>-1,-1+\lambda+\rho \in(-1,0),-1+\rho \in(-1,0) .
$$

Using the explicit resolutions $(14,15)$ we arrive at the following 2 -fold nestedBarnes integral (we also here have transformed $(s, t) \mapsto(2 s, 2 t)$ for notational convenience, and intentionally reverted back to "big-C" notation):

$$
C_{5, k}=-\frac{1}{240 \pi} \int_{2 \mathcal{C}_{s}} \int_{2 \mathcal{C}_{t}} d s d t \frac{\Gamma^{3}(s+(1+k) / 2) \Gamma^{3}(t-s)}{\Gamma(s+1+k / 2) \Gamma(t-s+1 / 2)} 4^{-t} \Gamma^{2}(-t) .
$$

It is of interest that another 2-dimensional integral - but evidently of markedly different character-was derivable for $C_{5}:=C_{5,1}$ in the separate treatment [8]. 


\subsection{Measure-theoretic representation}

Again starting from the original definition (1) we denote the sum of cosh terms as $U$, and develop a measure-theoretic form:

$$
C_{n, k}=\frac{1}{n !} \int_{n}^{\infty} \frac{d U}{U^{k+1}} \frac{\partial}{\partial U} \int_{\sum \cosh x_{k} \leq U} d x_{1} \cdots d x_{n},
$$

or, upon integration by parts,

$$
C_{n, k}=\frac{k+1}{n !} 2^{n+2} \int_{0}^{\infty} \frac{r V_{n}(r)}{\left(2 r^{2}+n\right)^{k+2}} d r
$$

where the volume $V_{n}$ is that of a "hyper-ellipsoid" of "radius" $r$ :

$$
V_{n}(r):=\int_{\sum \sinh ^{2} y_{k} \leq r^{2}} d y_{1} \cdots d y_{n}
$$

A test case is $n=1$, for which $V_{1}(r)=2 \operatorname{arcsinh} r$, and this measure-theoretic form agrees with (14).

This approach has not been taken further; however, note that we always have a 1-dimensional integral, for any $n$-an advantage shared by the Besselkernel representations. In the measure-theoretic case here, though, all involved functions are elementary. It is also interesting that if we had omniscience in regard to the properties of the hyper-ellipsoid, we would settle many questions about the $C_{n, k}$.

\subsection{An $n$-variant recursion and the elusive $C_{5}$}

Presumably the convolution Theorem 7 could be invoked, the resulting residue calculus giving us relations between the $c_{n, k}$ and entities $c_{p, j}$ with $p<n$. However there is a much more direct way to establish an $n$-variant recursion (i.e., now we have the first index $n$ changing on $c_{n, k}$ ). The Bessel-kernel representation (7) together with the insertion of one copy of $K_{0}$ in the form of an ascending series

$$
K_{0}^{(a s c)}(t)=\sum_{k \geq 0} \frac{t^{2 k}}{4^{k} k !^{2}}\left\{H_{k}-\gamma-\log (t / 2)\right\},
$$

see $[1,8]$, immediately yields an $n$-variant recursion (recall $c_{n, k}:=\Gamma(k+$ 1) $\left.2^{-n} n ! C_{n, k}\right)$ :

$$
c_{n, k}=\sum_{m \geq 0} \frac{1}{4^{m}} \frac{1}{m !^{2}}\left\{\left(H_{m}^{(1)}-\gamma+\log 2\right) c_{n-1, k+2 m}-c_{n-1, k+2 m}^{\prime}\right\},
$$

where the derivative is with respect to the second index, i.e. $c_{n, q}^{\prime}:=\partial c_{n, q} / \partial q$. Interestingly, for the problematic Ising integral $C_{5}:=C_{5,1}=c_{5,1} / 450$, we actually know all of the $c_{4,2 m+1}$ in principle, from Theorem 2 and the resulting algorithm. Unfortunately we still do not have a convenient representation for $c_{4,2 m+1}^{\prime}$, but at least we have derived a computational series involving, say, numerical differentiation, for $C_{5}$. 


\subsection{Bessel-moment relation}

Using an integration by parts, namely

$$
\frac{1}{\Gamma(k+1)} \int_{0}^{\infty} t^{k} K_{0}^{n}(t) d t=\frac{1}{\Gamma(k+3)} \int_{0}^{\infty} t^{k+2}\left(K_{0}^{n}(t)\right)^{\prime \prime} d t
$$

in the original definition (1), we can iterate in view of the recursion Conjecture 1 to write an equivalent conjecture as a Bessel-moment phenomenon-with $M:=$ $\lfloor(n+1) / 2\rfloor$ as in the conjecture:

$$
0 \stackrel{?}{=} \int_{0}^{\infty} t^{k+2 M}\left(P_{M}(k) K_{0}^{n}+P_{M-1}(k)\left(K_{0}^{n}\right)^{\prime \prime}+\cdots+P_{0}(k)\left(K_{0}^{n}\right)^{(2 M)}\right) d t .
$$

It is remarkable that polynomials $P_{0}, \ldots, P_{M}$ exist such that this moment integral appears to vanish for general complex $k \in \mathcal{K}$ (of course, the equivalent recursion relations are likewise remarkable). Note that the suspected vanishing of the above moment integral has been proven for $n=1,2,3,4$ and appropriate respective polynomials.

We have not taken this moment relation any further than to make the following observation. Using the asymptotic series [1]:

$$
K_{0}^{(a s y)}(t) \sim \sqrt{\frac{\pi}{2 t}} e^{-t} \sum_{m=0}^{\infty} \frac{(-1)^{m}((2 m) !)^{2}}{m !^{3}(32 t)^{m}}
$$

one may ask how the Bessel-moment integral above behaves when the asymptotic form is (naively, perhaps illegally) simply inserted into the integral. Surprisingly, if one truncates the sum (59) at a high enough $m$, and solves for the polynomials that minimize the $k$-degree of the moment integral, one evidently finds the correct polynomials exactly.

For example, we took the summation index $m$ up through 18, and solved symbolically for the higher powers of $k$ in the moment integral's result to vanish, and found we had detected this relation (36) previously, numerically, so it was pleasing to find the same polynomials via this admittedly nonrigorous handling of the moment integral. The fascinating nuance here is that, evidently, the recursion polynomials depend in some profound sense on the coefficients of the asymptotic expansion in (59).

\section{Extreme-precision numerics}

Using the Bessel-kernel representation (7), we have calculated to 500-digit accuracy values of $C_{n, k}$ for all integers $n, k$, where $n \in[2,12]$ and $k \in[0,25]$. This was done using the ARPREC arbitrary precision software [10] and the tanh-sinh quadrature scheme [11]. We have placed a listing of these numerical values on a website [9]. These were the raw data on which most of our discoveries were based. 


\section{Conclusion and open problems}

We wish to emphasize that the interaction of sophisticated numeric and symbolic computing has played an irreplaceable role in the work described herein. Indeed, we believe that these results would have been much more difficult, if not impossible, to deduce without reliance on heavy-duty computer power and sophisticated algorithms. Some of the techniques we employed include extreme-precision quadrature, PSLQ integer relation detection programs, generating function packages, high-accuracy least-squares polynomial fitting, and Wilf-Zeilberger theorem-proving software. We wish to thank those who have provided both the hardware and the software we have used. pelling.

We finish by recording some of the open problems we find the most com-

- While Conjectures 2 and 3 are probably out of current reach, what progress is possible on Conjecture 1. Specifically:

- How might one prove the conjectured recursion for $n=5$, from (35), using, say, the nested-Barnes representation (55)? This might amount to a higher-dimensional application of Wilf-Zeilberger methods [39].

- Is there a reasonable closed form for some or all of the following constants: $C_{5,1}, C_{4,0}, C_{3,2} / C_{3,0}, C_{4,2} / C_{4,0}$ ?

Acknowledgments. We are indebted to V. Adamchik, P. Paule, C. Schneider, P. Wellin, and A. van der Poorten. We give special acknowledgment to to W. Zudilin whose advice and expertise in various aspects of this research were indispensable.

\section{References}

[1] Milton Abramowitz and Irene A. Stegun, Handbook of Mathematical Functions, Dover, NY, 1970.

[2] V. Adamchik, "The evaluation of integrals of Bessel functions via $G$-function identities." Journal of Computational and Applied Mathematics, 64 (1995), 283-290.

[3] V. Adamchik, Private communication, Mar 2006.

[4] G. Almkvist and W. Zudilin, "Differential equations, mirror maps and zeta values," E-printmath.NT/0402386, 2004.

[5] R. Apéry, "Irrationalité de $\zeta(2)$ et $\zeta(3)$," Astérisque 61, (1979), 11-13.

[6] David H. Bailey and Jonathan M. Borwein, "Highly parallel, high-precision numerical integration," D-drive Preprint \#294, 2005. Also available at http://crd.lbl.gov/ dhbailey/dhbpapers/quadparallel.pdf. 
[7] David H. Bailey and Jonathan M. Borwein, "Effective error bounds for Euler-Maclaurin-based quadrature schemes," D-drive Preprint \#297, 2005. Also available at http://crd.lbl.gov/ dhbailey/dhbpapers/em-error.pdf.

[8] David H. Bailey, Jonathan M. Borwein and Richard E. Crandall, "Integrals of the Ising class," J. Phys. A.: Math. Gen., to appear (2007). Also, D-drive Preprint \#324, 2006, and http://crd.lbl.gov/ dhbailey/dhbpapers/ising.pdf.

[9] David H. Bailey, Jonathan M. Borwein and Richard E. Crandall, "Ising data," available at http://crd.lbl.gov/ ${ }^{d h b a i l e y / d h b p a p e r s / i s i n g-d a t a . p d f . ~}$

[10] David H. Bailey, Yozo Hida, Xiaoye S. Li and Brandon Thompson, "ARPREC: An Arbitrary Precision Computation Package," 2002, available at http://crd.lbl.gov/ dhbailey/dhbpapers/arprec.pdf.

[11] David H. Bailey, Xiaoye S. Li and Karthik Jeyabalan, "A comparison of three high-precision quadrature schemes," Experimental Mathematics, 14 (2005), $317-329$

[12] A. Bećirović et al., "Hypergeometric summation algorithms for high-order finite elements," Johann Kepler University, Linz. Technical Report \#2006-8.

[13] H. E. Boos and V. E. Korepin, "Quantum spin chains and Riemann zeta function with odd argument," Preprint, available at http://www . arxiv.org/hep-th/0104008, 2001.

[14] J.M. Borwein, "Hilbert Inequalities and Witten Zeta-functions," D-drive Preprint \#309, 2005.

[15] Jonathan M. Borwein and David H. Bailey, Mathematics by Experiment, AK Peters, 2003.

[16] Jonathan M. Borwein, David H. Bailey and Roland Girgensohn, Experimentation in Mathematics, AK Peters, 2004.

[17] Jonathan M. Borwein, David M. Bradley and Richard E. Crandall, "Computational strategies for the Riemann zeta function," Journal of Computational and Applied Mathematics, special volume "Numerical Analysis in the 20th Century. Vol. 1: Approximation Theory." 121 (2000), 247-296 [CECM Preprint 98:118].

[18] Jonathan M. Borwein and Peter B. Borwein, Pi and the AGM, John Wiley, 1987.

[19] D. Bowman and J. McLaughlin, "Polynomial continued fractions," Acta Arithmetica, 103 (2002), 329-342.

[20] P. Flajolet et al., "On the non-holonomic character of logarithms, powers, and the $n$-th prime function," Electronic Journal of Combinatorics, 11, \#A00, 2005.

[21] Frances Y. Kuo and Ian H. Sloan, "Lifting the curse of dimensionality," Notices of the AMS, 52, no. 11, (2005), 1320-1329. 
[22] L. Lewin, Polylogarithms and Associated Functions, North Holland, 1981.

[23] L. Lorentzen and H. Waadeland, Continued Fractions with Applications, North-Holland, Amsterdam, 1992.

[24] T. P. Martin, "Shells of Atoms," Phys. Reports, 273 (1996), 199-241.

[25] J-M. Maillard, Private communication, January 2005.

[26] J. McLaughlin and N. Wyshinski, "Real numbers with polynomial continued fraction expansions," Manuscript, 2004.

[27] B. Nickel, "On the singularity structure of the 2D Ising model susceptibility," Journal of Physics A: Mathematics and General, 32 (1999), 3889-3906.

[28] F. Oberhettinger, Tables of Fourier Transforms and Fourier Transforms of Distributions, Springer-Verlag, 1990.

[29] W. Orrick, B. Nickel, A. Guttmann, and J. Perk, "The susceptibility of the square lattice Ising model: New developments," Manuscript, March 2001.

[30] J. Palmer and C. Tracy, "Two-dimensional Ising correlations: Convergence of the scaling limit," Advances in Applied Mathematics, 2 (1981), 329-388.

[31] M. Prévost, "A new proof of the irrationality of $\zeta(2)$ and $\zeta(3)$ using Padé approximants," Journal of Computational and Applied Mathematics, 67 (1996), 219-235.

[32] K. Roach, "Meijer G-function representations," Proceedings of the 1997 International Symposium on Symbolic and Algebraic Computation, 205-211, New York, ACM, 1997.

[33] C. Schneider, Private communication, July 2006.

[34] C. A. Tracy, "Painlevé transcendents and scaling functions of the two-dimensional Ising model," in Nonlinear Equations in Physics and Mathematics, ed. A. O. Barut, D. Reidel Publ. Co., Dordrecht, Holland, (1978), $378-380$.

[35] C. Tracy, Private communication, Oct-Jan 2005.

[36] C. Tracy, Unpublished notes, 1976.

[37] A. J. van der Poorten and I. E. Shparlinski, "On linear recurrence sequences with polynomial coefficients," Manuscript, 2005.

[38] A. J. van der Poorten, "A proof that Euler missed: Apéry's proof of the irrationality of $\zeta(3)$," Mathematics Intelligencer, 1, no. 4 (1978/79), 195-203.

[39] H. Wilf and D. Zeilberger, "An algorithmic proof theory for hypergeometric (ordinary and "q") multisum/integral identities," Inventiones Mathematicae 108, 575-63, 1992.

[40] T. T. Wu, B. M. McCoy, C. A. Tracy and E. Barouch, "Spin-spin correlation functions for the two-dimensional Ising model: Exact theory in the scaling region," Physical Review B13 (1976), 316-374. 
[41] N. Zenine, S. Boukraa, S. Hassani and J-M Maillard, "Square lattice Ising susceptibility: Connection matrices and singular behaviour of $\chi^{(3)}$ and $\chi^{(4)}$," Journal of Physics A: Mathematical and General 38 (2005), 9439-9474.

[42] N. Zenine, S. Boukraa, S. Hassani and J-M Maillard, "Beyond series expansions: Mathematical structures for the susceptibility of the square lattice Ising model," J. Physics Conference (electronic review of J. Physics), to appear, June 2006.

[43] N. Zenine, S. Boukraa, S. Hassani and J-M Maillard, "Differential Galois groups of high order Fuchsian ODEs," Preprint, 2005.

[44] W. Zudilin, "An Apéry-like difference equation for Catalan's constant," Electronic Journal of Combinatorics 2003, \#R14, 1-10.

[45] W. Zudilin, "A third-order Apéry-like recursion for $\zeta(5)$," Preprint, available at http://www.arxiv.org/abs/math.NT/0206178, 2002.

[46] W. Zudlin, "An elementary proof of Apéry's theorem," Preprint, available at http://www.arxiv.org/math.NT/0202159, 2002.

[47] V. Zudilin, "Difference equations and the irrationality measure of numbers," Proceedings of the Steklov Institute of Mathematics, 218 (1997), 160-174.

[48] V. Zudilin, "Approximations to di- and tri-logarithms," Preprint, available at http://www . arxiv.org/math. NT/0409023, 2004.

[49] W. Zudilin, "Well-poised generation of Apéry-like recursions, Preprint, available at http://www.arxiv.org/math.NT/0307058, 2003.

[50] V. Zudilin, Private communication. 